\title{
Gyrofluid potential vorticity equation and turbulent equipartion states Paper
}

Madsen, Jens; Juul Rasmussen, Jens; Naulin, Volker; Nielsen, Anders Henry; Treue, Frederik

Published in:

Plasma Physics and Controlled Fusion

Link to article, DOI:

10.1088/0741-3335/57/5/054016

Publication date:

2015

Document Version

Peer reviewed version

Link back to DTU Orbit

Citation (APA):

Madsen, J., Juul Rasmussen, J., Naulin, V., Nielsen, A. H., \& Treue, F. (2015). Gyrofluid potential vorticity equation and turbulent equipartion states: Paper. Plasma Physics and Controlled Fusion, 57(5), [054016]. https://doi.org/10.1088/0741-3335/57/5/054016

\section{General rights}

Copyright and moral rights for the publications made accessible in the public portal are retained by the authors and/or other copyright owners and it is a condition of accessing publications that users recognise and abide by the legal requirements associated with these rights.

- Users may download and print one copy of any publication from the public portal for the purpose of private study or research.

- You may not further distribute the material or use it for any profit-making activity or commercial gain

- You may freely distribute the URL identifying the publication in the public portal 


\title{
Gyrofluid potential vorticity equation and turbulent equipartion states
}

\author{
J. Madsen, J. Juul Rasmussen, V. Naulin, A. H. Nielsen, F. \\ Treue \\ E-mail: jmad@fysik.dtu.dk \\ Department of Physics, Technical University of Denmark, DK-2800 Kgs. Lyngby, \\ Denmark
}

\begin{abstract}
An equation governing potential vorticity in a magnetized plasmas is derived. The equation is analogous to Ertel's theorem. In the long wave-length limit the potential vorticity equals the ratio of the gyro-frequency plus the $\boldsymbol{E} \times \boldsymbol{B}$ - and diamagnetic polarization densities to the particle density. The equation is relevant for transport barriers in magnetically confined plasmas because particle density, ion temperature and the radial electric field are mutually coupled through the potential vorticity. The potential vorticity equation is derived from an energy conserving, four-field, electrostatic, full-F gyrofluid model. It is shown that the gyrofluid model possesses two exact Lagrangian invariants. In systems where mixing uniformly distribute the Lagrangian invariants we derive the corresponding turbulent equipartion states. It is shown that the system is driven towards constant potential vorticity. Given particle density and magnetic field profiles we infer ion temperature and electric potential profiles from the derived turbulent equipartion states.
\end{abstract}

PACS numbers:

Submitted to: Plasma Phys. Control. Fusion 


\section{Introduction}

The concept of potential vorticity (PV) dates back to Ertel's theorem[1] which governs the time-evolution of PV. The theorem is an invaluable aid in understanding large scale fluid flows, particularly in planetary[2] and astrophysical fluid dynamics[3]. Analogues to Ertel's theorem in plasma physics exist $[4,5]$, but they have not had the same impact as Ertel's theorem. For ideal neutral fluids, PV is a Lagrangian invariant, i.e., it is conserved by each fluid element along its trajectory, and hence puts strong constraints on the dynamic evolution of the fluid. Highly relevant for magnetically confined plasmas are the applications of Ertel's theorem to systems with zonal flows in geophysical $[2,6,7,8]$ and astrophysical fluid dynamics[3]. By zonal flows we mean sheared banded flows. A prominent example[3] is the banded cloud pattern and east-west directed jets in the atmosphere of Jupiter. Common for these systems with zonal flows is the appearance of mixing in combination with non-constant PV profiles. In the context of PV dynamics, zonal flows are understood as a consequence of a stepwise homogenization of PV, forming a PV staircase. Each step forms a region of approximately constant PV, and the zonal flows are strongest in the vicinity of the interfaces between these regions of constant PV.

Similar ideas have been explored in plasma physics, where in particular turbulent equipartion (TEP) theory has been used to explain the formation of non-collisional density and temperature profiles, and the associated pinch velocities[9, 10, 11, 12, 13, 14]. In these theories Lagrangian invariants are mixed in regions bounded by closed streamlines. If the mixing is sufficiently strong, the existence of a small collisional diffusion homogenizes the Lagrangian invariants making them constant in the bounded regions. Turbulent equipartion theory has also been used to derive toroidal[15] and poloidal[16] momentum profiles and associated pinch velocities.

Here, we derive an equation analogues to Ertel's theorem governing the timeevolution of the magnetic-field-aligned PV from a full-F, electrostatic, 2D slab, collisionless gyrofluid model. In the long wave-length limit the PV reads

$$
\Pi \simeq \frac{\Omega_{i}+\omega^{*}}{n_{i}}
$$

where $\omega^{*}$ contains the magnetic-field-aligned $\boldsymbol{E} \times \boldsymbol{B}$ and diamagnetic vorticity, $n_{i}$ is the particle density and $\Omega_{i}$ is the ion gyro-frequency. Further, we identify two Lagrangian invariants of the model and derive two TEP states of the system. Interestingly, the first TEP state drives the system towards constant $\Pi$. Finally, we discuss the coupling between the radial electric field and the ion pressure prescribed by the TEP profile, in relation to zonal flows and transport barriers in magnetically confined plasmas, and analogies to PV stair-cases in geophysical and astrophysical fluid dynamics.

The remainder of the paper is organized as follows. In section 2 we present the full-F gyrofluid model used in this paper. A PV equation is presented in section 3 . In section 4 TEP states and the associated profiles are presented. In section 5 we discuss the results in relation to transport barrier physics. Finally, we sum up the results in 
section 6 .

\section{Model}

In this work we shall adopt a gyrofluid model[17] derived from full-F gyrokinetic[18] equations. Since we address the interplay between low-frequency turbulence and profiles, the full-F gyrofluid model is a good choice because it is global in the sense that no distinctions between fluctuations and background profiles are made. The model is fully non-linear and hence can describe micro-turbulence with gradient length-scales comparable to the gyro-radius. Furthermore, for algebraic manipulation gyrofluid models have an advantage over other low-frequency fluid models[19, 20] because the gyro-viscous cancellations $[21,22]$ appear naturally. In gyrofluid models the cancellation is carried out at the gyrokinetic level, which implies relatively simple gyrofluid model equations. The simplicity of gyrofluid models is striking because FLR-corrected drift fluid models can be recovered from gyrofluid models[23, 24, 17, 25].

Here, we restrict our analysis to a simple, electrostatic plasma in a $2 \mathrm{D}$ slab perpendicular to the magnetic field. Collisions and dynamics parallel to the magnetic field are neglected. Ions are described by the gyro-center density $N_{i}$ and the perpendicular gyro-center pressure $P_{i \perp}$, whose time-evolutions are governed by

$$
\begin{aligned}
& \frac{\partial}{\partial t} N_{i}+\nabla \cdot\left(N_{i}\left[\boldsymbol{u}_{E}+\boldsymbol{u}_{T}+\boldsymbol{u}_{\eta}\right]\right)=0, \\
& \frac{\partial}{\partial t} P_{i \perp}+\nabla \cdot\left(P_{i \perp}\left[\boldsymbol{u}_{E}+2 \boldsymbol{u}_{T}+2 \boldsymbol{u}_{\eta}\right]\right)-P_{i \perp}\left(\boldsymbol{u}_{E}+2 \boldsymbol{u}_{\eta}\right) \cdot \nabla \ln B=0,
\end{aligned}
$$

where the ion gyrofluid velocities are

$$
\boldsymbol{u}_{E}=\frac{\hat{\boldsymbol{b}} \times \nabla \psi}{B}, \quad \boldsymbol{u}_{T}=T_{i \perp} \frac{\hat{\boldsymbol{b}} \times \nabla \ln B}{q_{i} B}, \quad \boldsymbol{u}_{\eta}=\Gamma_{2} \phi \frac{\hat{\boldsymbol{b}} \times \nabla \eta}{B} .
$$

The perpendicular gyro-center temperature is defined as $T_{i \perp}=P_{i \perp} / N_{i}$ and $B$ denotes the magnetic field strength. The generalized potential

$$
\psi=\Gamma_{1} \phi-\frac{m\left|\nabla_{\perp} \phi\right|^{2}}{2 q_{i} B^{2}}
$$

entering the $\boldsymbol{E} \times \boldsymbol{B}$-drift $\boldsymbol{u}_{E}$ includes the gyro-averaged electrostatic potential $\Gamma_{1} \phi$, and the $\boldsymbol{E} \times \boldsymbol{B}$-energy which is important for momentum and energy conservation. The gyroaverage operator $\Gamma_{1}=1+\rho^{2} / 2 \nabla_{\perp}^{2}+\cdots$, is the gyrofluid moment of the gyrokinetic gyroaveraging operator, where $\rho^{2}=T_{i \perp} /\left(m_{i} \Omega_{i}^{2}\right)$ is the non-constant, thermal gyro-radius. $\boldsymbol{u}_{T}$ is the gyrofluid representation of the $\nabla B$-drift and $\boldsymbol{u}_{\eta}$ describes corrections to the

gyro-averaged electric potential by the second gyro-average operator $\Gamma_{2}=T_{\perp} \frac{\partial \Gamma_{1}}{\partial T_{\perp}}$ due to spatial variations of temperature and magnetic field strength $\nabla \eta=\nabla \ln B-\nabla \ln T_{i \perp}$.

As a consequence of the smallness of the electron to ion mass ratio, only the lowest order terms in the electron gyro-center coordinate transformation are retained, which corresponds to neglect of electron finite Larmor radius and finite electron inertia effects. Therefore, the electron gyrofluid moment variables are identical to the corresponding 
fluid moment variables e.g., $N_{e}=n_{e}$. The electron equations are

$$
\begin{aligned}
& \frac{\partial}{\partial t} n_{e}+\nabla \cdot\left(n_{e}\left[\boldsymbol{v}_{E}+\boldsymbol{v}_{T}\right]\right)=0, \\
& \frac{\partial}{\partial t} p_{e \perp}+\nabla \cdot\left(p_{e \perp}\left[\boldsymbol{v}_{E}+2 \boldsymbol{v}_{t}\right]\right)-p_{e \perp} \boldsymbol{v}_{E} \cdot \nabla \ln B=0,
\end{aligned}
$$

where

$$
\begin{aligned}
& \boldsymbol{v}_{E}=\frac{\hat{\boldsymbol{b}} \times \nabla \phi}{B}, \\
& \boldsymbol{v}_{t}=-t_{e \perp} \frac{\hat{\boldsymbol{b}} \times \nabla \ln B}{e B},
\end{aligned}
$$

are the electron $\boldsymbol{E} \times \boldsymbol{B}$-drift and $\nabla B$-drift, respectively, and the perpendicular electron temperature is $t_{e \perp}=p_{e \perp} / n_{e}$. In passing we note that the diamagnetic cancellations in equations (5) and (6) appeared automatically. In order to emphasize the fundamental difference between particle and gyrofluid moments, all standard particle fluid moments are written in lower case letters (e.g. $n_{i}, n_{e}, p_{i}, p_{e}, t_{i \perp}, t_{e \perp}$ ) whereas gyrofluid moments are written in upper case letters (e.g. $\left.N_{i}, P_{i \perp}, T_{i \perp}\right)$.

Electrons and ions are coupled through the quasi-neutrality constraint. By expressing the ion particle density $n_{i}$ in terms of gyrofluid moment variables we get

$$
n_{e}=n_{i}=\Gamma_{1}^{\dagger} N_{i}+\nabla \cdot\left(\frac{N_{i}}{\Omega_{i} B} \nabla_{\perp} \phi\right) .
$$

The operator $\Gamma_{1}^{\dagger}=1+\nabla_{\perp}^{2} \rho^{2} / 2+\cdots$ is the Hermitian conjugate operator to $\Gamma_{1}$. Note that

the Laplacian in $\Gamma_{1}^{\dagger}$ operates on $\rho^{2}$. This equation is also referred to as the polarization equation due to the explicit appearance of the polarization density, the last term on the right hand side, which is the manifestation of the polarization drift in gyrokinetic models[18] or equivalently the divergence of the polarization drift current $[24,26,25]$ in drift fluid models.

The total conserved energy for this system is

$$
\mathcal{E}=\int d^{3} \boldsymbol{r}\left(\frac{m_{i} N_{i}\left|\nabla_{\perp} \phi\right|^{2}}{2 B^{2}}+P_{i \perp}+p_{e \perp}\right)
$$

where boundary terms were discarded.

\section{Potential vorticity equation}

In neutral fluid dynamics Ertel's theorem[1] is an indispensable aid, particularly in geophysical and planetary fluids, for comprehension of the character and physics of a wide range of phenomena. By combining the fluid vorticity and density equations we get an equation

$$
\frac{d}{d t} \Pi=\frac{\boldsymbol{\omega}}{n} \cdot \nabla S+\nabla \lambda \cdot \frac{\nabla n \times \nabla p}{n^{3}}+\frac{\nabla \lambda}{n} \cdot \nabla \times \frac{\boldsymbol{F}}{n}
$$

describing the time evolution of $\mathrm{PV}: \Pi=\boldsymbol{\omega} \cdot \nabla \lambda / n$. Here, $n$ denotes the neutral fluid density, $\boldsymbol{\omega}=\nabla \times \boldsymbol{u}$ is the vorticity associated with the velocity field $\boldsymbol{u}, d / d t=\partial / \partial t+\boldsymbol{u} \cdot \nabla$ is the material derivative, $\boldsymbol{F}$ is the frictional force, and $\lambda$ is some scalar satisfying

$$
d \lambda / d t=S,
$$


where $S$ is a source. Ertels theorem states that if: i) $\lambda$ is a conserved quantity, ii) the fluid is either barotropic (i.e. the baroclinic vector vanishes: $\nabla n \times \nabla p=0$ ) or if $\lambda$ is solely a function of $n$ and $p$, and iii) the friction force $\mathrm{F}$ is negligible, then $\mathrm{PV}$ is a Lagrangian invariant, i.e. conserved in the frame moving with the velocity $\boldsymbol{u}$.

For a system steadily rotating with angular velocity $\Omega$ the PV becomes $\Pi=$ $\left(2 \boldsymbol{\Omega}+\boldsymbol{\omega}_{r}\right) \cdot \nabla \lambda / n$, where $\boldsymbol{\omega}_{r}$ is the relative vorticity measured in the rotating frame of reference.

To demonstrate the power of the concept of PV, we consider an example from oceanography. Large-scale flows in the ocean are well described by shallow water models[2]. Furthermore, frictional forces are negligible and the fluid is nearly barotropic. Therefore, any conserved fluid scalar $\lambda$ implies conservation of a corresponding potential vorticity. Since vertical mixing of the water column is negligible, the relative position of a fluid parcel $\lambda=\left(z-h_{B}\right) / H$ over the ocean bottom at $h_{B}$ in a fluid column of height $H$ is a Lagrangian invariant which implies that

$$
\frac{d}{d t} \frac{f+\omega}{H n}=0
$$

is a Lagrangian invariant. $f=2 \Omega \sin \theta$ is the Coriolis parameter describing the component of the planetary vorticity $2 \Omega$ normal to the ocean surface at latitude $\theta$ ( $f=0$ at equator). Since the density $n$ can be considered constant PV conservation tightly couples the local vorticity of the water column $\omega$, the height $H$, and the local component of the planetary vorticity $f$. Phenomena such as the spin-up of water columns when moving from shallow to deeper water and the path of the Gulf stream through the Atlantic Ocean can be explained by the concept of PV conservation in shallow water models.

Here we will show that the ion gyro-center density equation (1) provides an analogy to Ertel's theorem. For low ion temperature a plasma fluid analogue is readily derived from the ion equations of motion showing that $\left(\Omega_{i}+\boldsymbol{\omega}\right) / n$ is conserved in the inviscid limit when the baroclinic contribution vanishes[4]. Our work considers the case of nonzero ion temperature. In order to obtain the gyrofluid PV equation we introduce the following drifts

$$
\boldsymbol{u}_{\Omega}=T_{i \perp} \frac{\hat{\boldsymbol{b}} \times \nabla\left(\frac{N \Gamma_{2} \phi}{T_{i \perp}}\right)}{N_{i} B}, \quad \boldsymbol{u}_{D}=\frac{\hat{\boldsymbol{b}} \times \nabla P_{i \perp}}{q N_{i} B} .
$$

The divergences of the corresponding fluxes equal the original terms in equation (1)

$$
\nabla \cdot\left(N_{i} \boldsymbol{u}_{T}\right)=\nabla \cdot\left(N_{i} \boldsymbol{u}_{D}\right), \quad \nabla \cdot\left(N_{i} \boldsymbol{u}_{\eta}\right)=\nabla \cdot\left(N_{i} \boldsymbol{u}_{\Omega}\right) .
$$

Using these relations the gyro-center density equation (1) can be recast as

$$
\frac{d^{*}}{d t} \ln \left(\frac{\Omega_{i}}{N_{i}}\right)=\hat{\boldsymbol{b}} \cdot \frac{\nabla \ln \frac{N_{i}}{T_{i \perp}} \times \nabla T^{*}}{q B},
$$

where we have defined the material derivative and the modified temperature

$$
\frac{d^{*}}{d t}=\frac{\partial}{\partial t}+\left[\boldsymbol{u}_{E}+\boldsymbol{u}_{D}+\boldsymbol{u}_{\Omega}\right] \cdot \nabla, \quad T^{*}=T_{\perp}+q \Gamma_{2} \phi
$$


respectively. To see that equation (14) in fact describes the time-evolution of PV we express the ion gyro-center density $N_{i}$ in terms of particle density $n_{i}$, perpendicular pressure $p_{i \perp}$, and the electrostatic potential $\phi$ by solving equation (9) for $N_{i}$. In the long wavelength limit (LWL) $k_{\perp}^{4} \rho^{4} \ll 1$, where $k_{\perp}$ is the typical inverse perpendicular gradient length scale, we obtain the following expression for the gyro-center density

$$
N_{i} \simeq n_{i}\left(1-\frac{\omega^{*}}{\Omega_{i}}\right)
$$

where

$$
\omega^{*}=\frac{\Omega_{i}}{n_{i}}\left[\nabla_{\perp}^{2}\left(\frac{p_{i \perp}}{2 m \Omega_{i}^{2}}\right)+\nabla \cdot\left(\frac{n_{i}}{B \Omega_{i}} \nabla_{\perp} \phi\right)\right] .
$$

The last term in equation (17) equals the component of the $\boldsymbol{E} \times \boldsymbol{B}$-drift vorticity $\hat{\boldsymbol{b}} \cdot \nabla \times \boldsymbol{u}_{E}=\nabla \cdot\left(B^{-1} \nabla_{\perp} \phi\right)$ aligned with the magnetic field plus a cross-term $\nabla \ln \left(n_{i} / \Omega_{i}\right) \cdot \nabla_{\perp} \phi / B$. The first term in equation (17) equals half the magnetic-fieldaligned component of the diamagnetic vorticity $1 / 2 \hat{\boldsymbol{b}} \cdot \nabla \times \boldsymbol{u}_{D}=1 / 2 \nabla \cdot\left(\nabla_{\perp} p_{i} /\left(q n_{i} B\right)\right)$ plus a cross-term $1 / 2\left(q n_{i} B\right)^{-1} \nabla_{\perp} p_{i} \cdot\left[\nabla \ln n_{i}+3 \nabla \Omega_{i}\right]$. Only half the diamagnetic vorticity appears which is a consequence of the underlying gyro-viscous cancellation which to lowest order annihilates the diamagnetic advection of momentum and thereby breaks the symmetry in the inertia term. Since $\omega^{*} / \Omega_{i}$ is small:

$$
\frac{\Omega_{i}}{N_{i}} \simeq \frac{\Omega_{i}+\omega^{*}}{n_{i}}
$$

and hence equation (14) becomes

$$
\frac{d^{*}}{d t} \ln \left(\frac{\Omega_{i}+\omega^{*}}{n_{i}}\right)=\hat{\boldsymbol{b}} \cdot \frac{\nabla \ln \frac{N_{i}}{T_{i \perp}} \times \nabla T^{*}}{q B} .
$$

Identifying $\hat{\boldsymbol{b}}$ as $\nabla \lambda$ we note the strong resemblance of this equation to Ertel's equation (11).

To sum up, the electrostatic, 2D slab geometry gyrofluid PV equation (14) implies that in regions where (i) viscous forces, external forces and parallel dynamics can be neglected and (ii) when the right hand side - the modified baroclinic vector - vanishes, $\Omega_{i} / N$ is a Lagrangian invariant. This $\mathrm{PV}$ theorem is important for, e.g., zonal flow generation because it links the electric field to the density and temperature profiles through equation (16). We will discuss this further in section 5.

\section{Lagrangian invariants and turbulent equipartition profiles}

We will now identify two quantities that are conserved along specific fluid trajectories. These Lagrangian invariants are derived by combining the gyrofluid PV equation (14) with a recasted version of the perpendicular gyro-center pressure equation (2). First, we use the identities

$$
\begin{aligned}
& \nabla \cdot\left(P_{i \perp} \boldsymbol{u}_{T}\right)=\nabla \cdot\left(P_{i \perp} \boldsymbol{u}_{D}\right)+P_{i \perp} \nabla T_{i \perp} \cdot \frac{\hat{\boldsymbol{b}} \times \nabla \ln B}{q B}-\nabla T_{i \perp} \cdot \frac{\hat{\boldsymbol{b}} \times \nabla P_{i \perp}}{q B}, \\
& \nabla \cdot\left(N_{i} \boldsymbol{u}_{\eta}\right)=\nabla \cdot\left(P_{i \perp} \boldsymbol{u}_{\Omega}\right)+N_{i} \Gamma_{2} \phi \nabla T_{i \perp} \cdot \frac{\hat{\boldsymbol{b}} \times \nabla \ln B}{B}-\nabla T_{i \perp} \cdot \frac{\hat{\boldsymbol{b}} \times \nabla\left(N \Gamma_{2} \phi\right)}{B}
\end{aligned}
$$


to write equation (2) as

$$
\frac{d^{*}}{d t} \ln \frac{P_{i \perp}}{N_{i}^{2}}=-2 \frac{\hat{\boldsymbol{b}} \times \nabla T^{*}}{q B} \cdot \nabla \ln \frac{N_{i}}{B},
$$

where the material derivative and the generalized temperature $T^{*}$ are defined in equation (15). The symmetry of equation (14) and equation (22) suggests[10] the Lagrangian invariants to be on the following form:

$$
\nabla L=\nabla\left[\alpha \ln \frac{N_{i}}{B}+\ln \frac{P_{i \perp}}{N_{i}^{2}}\right]
$$

where $\alpha$ is scalar. An equation for $L$ is obtained by combining equations (14) and (22), which can be solved for $\alpha$ requiring that $L$ is a Lagrangian invariant. Two solutions $\alpha= \pm \sqrt{2}$ are obtained corresponding to the exact Lagrangian invariants

$$
\nabla L_{ \pm}=\nabla\left[ \pm \sqrt{2} \ln \frac{N_{i}}{B}+\ln \frac{P_{i \perp}}{N_{i}^{2}}\right]
$$

conserved along orbits determined by

$$
\left(\frac{d^{*}}{d t}+\boldsymbol{u}_{ \pm} \cdot \nabla\right) L_{ \pm}=0
$$

where

$$
\boldsymbol{u}_{ \pm}= \pm \sqrt{2} \frac{\hat{\boldsymbol{b}} \times \nabla T_{i}^{*}}{q B}
$$

The electron fluid also possesses Lagrangian invariants which can be directly derived from the governing equations (5) and (6) or alternatively by evaluating equation (25) in the limit of small gyro-radius. Either way, the electron invariants are

$$
\nabla l_{ \pm}=\nabla\left[ \pm \sqrt{2} \ln \frac{n_{e}}{B}+\ln \frac{p_{e \perp}}{n_{e}^{2}}\right]
$$

which are conserved along trajectories with velocities

$$
\boldsymbol{v}=\boldsymbol{v}_{E}-\frac{\hat{\boldsymbol{b}} \times \nabla p_{e \perp}}{e n_{e} B} \mp \sqrt{2} \frac{\hat{\boldsymbol{b}} \times \nabla T_{e \perp}}{e B} .
$$

\subsection{Turbulent equipartion profiles}

Regardless of whether the flow is laminar or turbulent, $L_{ \pm}$and $l_{ \pm}$are Lagrangian invariants. In either case, if in a bounded region the mixing time-scale is faster than other competing time-scales (e.g., collisional diffusion time-scale), the Lagrangian invariants will be uniformly distributed, a state denoted turbulent equipartition (TEP) [9, 27]. A simple example is the equipartition of $n / B$ in simplified 2D drift-fluid turbulence[28, 12], which implies that the particle density profile is given by the magnetic field $n \sim B$ and that $n$ is transported up-gradient by the so-called curvature pinch velocity[27].

Here, we are concerned with mixing of the Lagrangian invariants $L_{+}$and $L_{-}$given in equation (24). An equipartion of $L_{+}$and $L_{-}$due to turbulent mixing implies that profiles are driven towards

$$
\frac{N_{i}}{B}=\text { const. }, \quad \frac{T_{i \perp}}{B}=\text { const. }
$$


Interestingly, the $N_{i} \propto B$ TEP profile drives the system towards constant PV, whose time-evolution is given in equation (14). To further investigate the TEP state, it is instructive to express the PV and therefore the ion gyro-center density in terms of measurable quantities. Since we are concerned with profiles inferred from the TEP state, we take the ion gyro-center density in the LWL as in equation (18) which allows us to express the $N_{i} \propto B$ TEP profile as

$$
\frac{\Omega_{i}+\omega^{*}}{n_{i}} \simeq \text { const. }=c_{1}
$$

where $\omega^{*}$ is defined in equation (17). In order to express the TEP $T_{i \perp} \propto B$ profile in terms of measurable quantities, a relation similar to the quasi-neutrality constraint equation (9) for the perpendicular ion particle pressure $p_{i \perp}$ is needed. Using the same procedures[17] which led to equation (9) we obtain the following LWL approximation for the perpendicular ion gyro-center pressure

$$
P_{i \perp}=p_{i \perp}-p_{i}^{*}, \quad p_{i}^{*}=\nabla_{\perp}^{2}\left(\frac{t_{i \perp} p_{i \perp}}{m_{i} \Omega_{i}^{2}}\right)+2 \nabla \cdot\left(\frac{p_{i \perp}}{B \Omega_{i}} \nabla_{\perp} \phi\right)
$$

and hence that the profiles in the LWL are driven towards

$$
\frac{c_{1}\left(p_{i \perp}-p_{i}^{*}\right)}{B \Omega} \simeq \text { const. }=c_{2},
$$

where equation (30) was inserted.

Equations (30) and (32) form a coupled set of ordinary differential equations. For example if the particle density $n$ and the magnetic field strength $B$ are specified, the electric potential $\phi$ and the perpendicular ion temperature $t_{i \perp}$ can be determined given appropriate boundary conditions and values of the constants $c_{1}$ and $c_{2}$. The TEP states therefore couple the particle density, the ion temperature, the magnetic field and the electric field profiles. To illustrate this point we consider the special case where $\Omega / n=C$ is constant. In that situation $\omega^{*} / \Omega$ is also constant, and hence equations (30) and (32) reduce to the coupled equations

$$
\begin{array}{ll}
\frac{d}{d x} E & =\frac{d^{2}}{d x^{2}}\left(\frac{t_{i \perp}}{2 m_{i} \Omega_{i}}\right)-\Omega_{i} C\left(c_{1}-C\right), \\
\frac{d}{d x}\left(\frac{E t_{i \perp}}{B}\right) & =\frac{c_{2}}{2 C c_{1}}-\frac{t_{i \perp} \Omega_{i}}{2}+\frac{1}{2} \frac{d^{2}}{d x^{2}}\left(\frac{t_{i \perp}}{m_{i} \Omega_{i}}\right),
\end{array}
$$

where $E=-\frac{d}{d x} \phi$ denotes the electric field, and $x$ is the "radial" slab coordinate antiparallel to the magnetic field gradient. In this special case the coupling of the electric field and the ion temperature is evident. We will discuss this coupling further in the next section.

Lastly, a uniform distribution of the electron fluid Lagrangian invariants $l_{+}$and $l_{-}$ due to turbulent mixing implies that the electron profiles are driven towards

$$
\frac{n_{e}}{B}=\text { const. }, \quad \frac{t_{e \perp}}{B}=\text { const. }
$$

as was also found in Ref. [27]. The result obtained here can easily be derived for isotropic electron temperature $t_{e \perp}=t_{e \|}$. 
We would like to emphasize that profiles predicted by TEP theory must be used with care because ions and electrons are coupled. Especially, if the ion TEP states are used to infer the electric field profile without considering the electrons, as is possible using equations (30) and (32). However, it is easily imaginable that ion and electron TEP states are not simultaneously reached, e.g., if their particle, momentum and heat sinks and sources are different. Given that the ion and electron profiles are simultaneously driven towards their corresponding TEP states, the ion profiles in equation (29) reduce to

$$
\frac{1}{B} \frac{d}{d x}\left(\frac{E}{B}\right)=\text { const. }, \quad \frac{t_{i \perp}}{B}+\frac{2}{B^{2} \Omega_{i}} E \frac{d}{d x} t_{i \perp}=\text { const. }
$$

respectively. In this state the shearing rate of the radial electric field follows the magnetic field with a variation constrained by the ion temperature profile.

\section{Discussion}

In a magnetically confined fusion plasma we cannot expect PV nor the Lagrangian invariants in equations (29) and (35) to be globally mixed and hence uniformly distributed over the entire plasma from the core to the wall. Due to the presence of spatially localized sinks and sources, the PV profile is expected to be non-constant. Nonetheless, we speculate that the plasma can be organized such that the Lagrangian invariants are constant in bounded regions. A stepwise homogenization has been observed in geophysical[7, 6] and astrophysical[3] systems, where strong zonal flows near the interfaces between the regions of constant PV have been observed. We hypothesize that similar mechanisms involving homogenization of PV could be relevant for explaining the large scale flows associated with transport barriers in magnetically confined plasmas.

In order to illustrate this hypothesis, we have inferred the ion temperature and the electric field profiles by solving the coupled boundary value problems in equations (30) and (32) for given profiles of the particle density $n_{i}(x)$, the magnetic field $B(x)$, and the TEP constants $c_{1}(x)$ and $c_{2}(x)$. We show two examples to illustrate how the constants $c_{1}$ and $c_{2}$ influence the solutions. In both examples the particle density profile is a monotonically decreasing function with a localized steep gradient pedestal region. The boundary value problems were solved numerically using the bvp4c Matlab routine[29]. All quantities are Bohm normalized:

$$
x / \rho_{i 0} \rightarrow x, \quad \tau \Omega_{i 0} \rightarrow \tau, \quad e \phi / t_{i 0} \rightarrow \phi, \quad t_{i \perp} / t_{i 0} \rightarrow t_{i \perp}, \quad n_{i} / n_{i 0} \rightarrow n_{i}
$$

where $t_{i 0}=t_{i \perp}(x=0), \Omega_{i 0}$ is the ion gyro-frequency at $x=0$, and $\rho_{i 0}=\Omega_{i 0}^{-1} \sqrt{t_{i 0} / m_{i}}$. In both examples the equations were solved on a domain $0 \leq x \leq 100$, and we applied the boundary conditions

$$
t_{i \perp}(0)=1, \quad \frac{d}{d x} t_{i \perp}(100)=0, \quad \phi(0)=\phi(100)=0 .
$$

Example 1 -stepwise homogenization. As shown in figure 1 the particle density is proportional to the magnetic field except near $x=50$, where the value of the particle 
density strongly decreases. $c_{1}$ and $c_{2}$ are chosen such that they follow the background
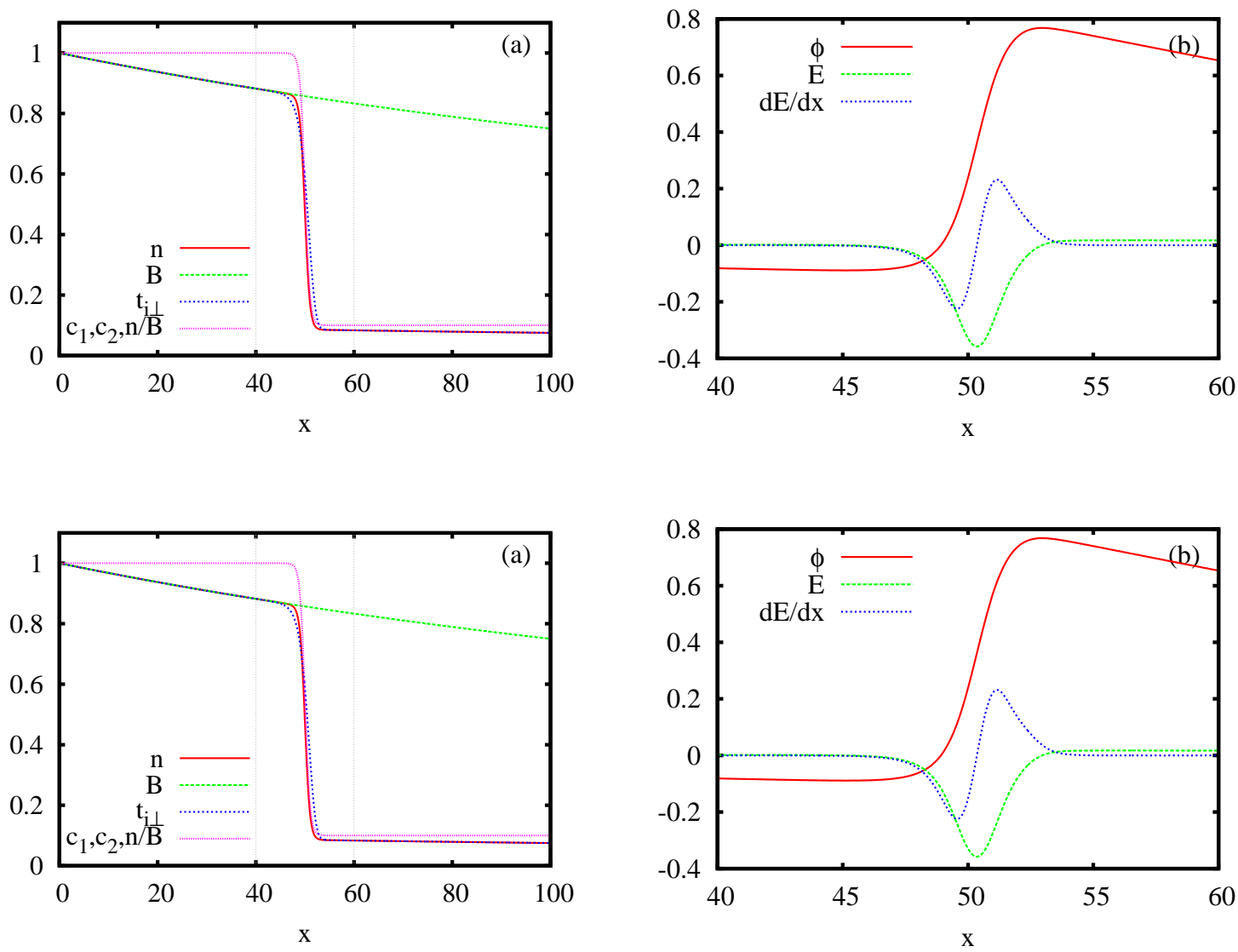

Figure 1. Input profiles of (a) particle density $n$, magnetic field strength $B$, TEP states $c_{1}$ and $c_{2}$, and $n / B$. Vertical grey lines indicate the domain plotted in (b). Numerical solutions to the coupled boundary value problems given in equations (30) and (32) for (a) $t_{i \perp}$ and (b) the electric potential $\phi$, also showing the electric field $E$ and the shearing rate $d E / d x$.

value of the $N \propto B$ TEP state defined in equation (30) where $\omega^{*} \simeq 0$. Since $n_{i}$ is proportional to $B$ away from $x=50$ we get that $c_{1}(x)=c_{2}(x)=n_{i}(x) / B(x)$. As shown in figure 1 (a), $c_{1}$ and $c_{2}$ are therefore stepwise constant. The solutions $t_{i \perp}$ and $\phi$ are shown in figure 1(a) and 1(b), respectively. Outside the steep gradient region the ion temperature coincides with the particle density profile and is therefore proportional to the magnetic field. Also, the electric field goes towards zero outside the steep region. Since $n_{i} \propto B$ in these regions it is not surprising that $t_{i}$ and $\phi$ are solutions to equations (33)-(34). Near $x=50$ we observe that the ion temperature strongly decreases as has a negative slope comparable to the particle density gradient but slightly less steep. The electric field is negative and is strongly sheared forming a well centered around $x \simeq 50$.

Example 2 - TEP state with particle density pedestal. Another possible scenario is that profiles are driven towards the TEP states given in equations (30) and (32) across 
a particle density pedestal region. In this case the TEP state constants are fixed to the mean value of $n / B: c_{1}=c_{2}=0.98$. The solutions $t_{i \perp}$ and $\phi$ to the coupled boundary value problems given in equation (30) and (32) are shown in figure $2(\mathrm{a})$ and 2 (b), respectively. The ion temperature profile decreases in the vicinity of the particle
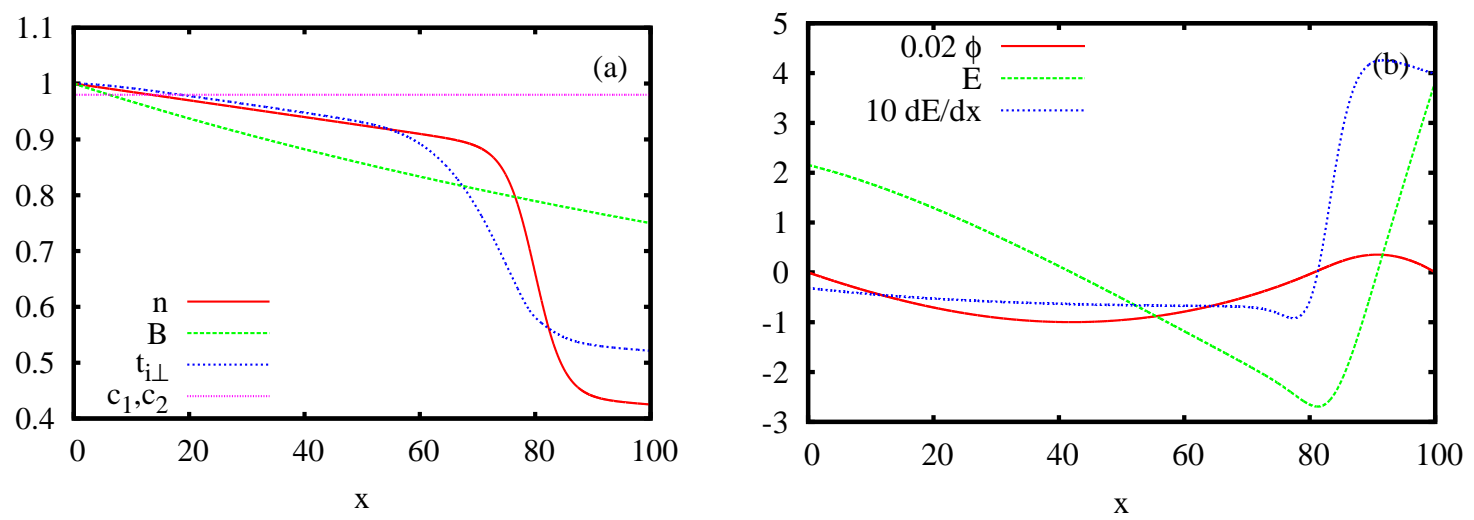

Figure 2. Input profiles of (a) particle density $n$, magnetic field strength $B$, and TEP states $c_{1}$ and $c_{2}$. Numerical solutions to the coupled boundary value problems given in equations (30) and (32) for (a) $t_{i \perp}$, and (b) the electric potential $\phi$, also showing the electric field $E$ and the shearing rate $d E / d x$.

density pedestal region, but the ion temperature profile is less steep than the particle density profile. In the region to the left of the particle density pedestal region the electric field is approximately a linearly decreasing function, and hence with a constant shearing rate. When approaching the pedestal region the electric field rapidly increases and forms a well with a strong shear. We note that these features in ion temperature and electric field profiles are qualitatively in agreement with experimental observations of sheared flows associated with transport barriers[30].

Another possible scenario is the combined ion and electron TEP state given in equation (36). In this state the $\boldsymbol{E} \times \boldsymbol{B}$-shearing rate is constant. Regions with constant shearing rates on each side of a transport barrier were observed in the $\mathrm{H}-1$ device[31]. In future work we will compare our findings with numerical simulations and experimental measurements.

\section{Summary}

We have derived a non-zero ion temperature potential vorticity equation analogous to Ertel's theorem for a plasma in a $2 \mathrm{D}$ slab. The potential vorticity equation (14) is derived from an electrostatic, inviscid, full-F gyrofluid turbulence model, which is fully non-linear and makes no distinction between fluctuations and profiles. We show that when evaluated in the long wave-length limit the potential vorticity equals the ratio of the ion gyro-frequency plus the $\boldsymbol{E} \times \boldsymbol{B}$ and diamagnetic polarization densities to the particle density. We show that the gyrofluid model possesses two Lagrangian invariants which are used to derive two turbulent equipartition states given in equation 
(29). In the turbulent equipartion state profiles are driven towards constant potential vorticity. The turbulent equipartition states are evaluated in the long wave-length limit revealing that the turbulent equipartion states couple particle density, temperature, and the electric field. We hypothesize that the results are important for zonal flows and the associated transport barriers in magnetically confined plasmas, drawing analogies to potential vorticity staircases in geophysical and astrophysical fluid dynamics. To support this hypothesis we infer the ion temperature and electric potential profiles from the turbulent equipartion states given profiles of the particle density and the magnetic field. The inferred electric field, shown in figures 1 and 2, is strongly sheared and forms a well in regions where the particle density profiles has steep gradients, and hence qualitatively share features with the observed electric field associated with transport barrier in magnetically confined plasmas.

\section{Acknowledgments}

The initial phase of this work was supported by an EFDA fusion researcher fellowship (WP11-FRF-RISOE/MADSEN). One of the authors (JJR) acknowledge fruitful discussions on geophysical potential vorticity dynamics at the 2014 KITP programme on "Wave-Flow Interaction in Geophysics, Climate, Astrophysics and Plasmas" at University of California, Santa Barbara USA.

\section{References}

[1] Ertel H 1942 Meteorol. Z. 59277

[2] Pedlosky J 1987 Geophysical fluid dynamics. Springer-Verlag New York

[3] Marcus P and Shetty S 2011 Philosophical Transactions of the Royal Society A: Mathematical, Physical and Engineering Sciences 369771

[4] Weiland J 2000 Collective modes in inhomogeneous plasma : kinetic and advanced fluid theory. Plasma physics series. Institute of Physics, Bristol (UK), Philadelphia

[5] Horton C W and Ichikawa Y H 1996 Chaos and Structures in Nonlinear Plasmas. World Scientific

[6] Phillips O M 1972 Deep-Sea Research 19797

[7] Rhines O M and Young W R 1982 J. Fluid Mech. 122347

[8] Dritschel D G and McIntyre M E 2008 Journal of the Atmospheric Sciences 65855

[9] Yankov V V 1994 JETP Lett. 60171

[10] Isichenko M B and Yankov V V 1997 Physics Reports 283161

[11] Yankov V V and Nycander J 1997 Phys.Plasmas, 42907

[12] Naulin V, Nycander J, and Rasmussen J Juul 1998 Phys. Rev. Lett. 814148

[13] Naulin V, Rasmussen J Juul, and Nycander J 2003 Phys. Plasmas 101075

[14] Garbet X et al 2003 Phys. Rev. Lett. 91035001

[15] Hahm T S et al 2008 Phys. Plasmas 15055902

[16] McDevitt C J et al2010 Phys. Plasmas, 17112509

[17] Madsen J 2013 Phys. Plasmas, 20072301

[18] Brizard A J and Hahm T S 2007 Rev. Mod. Phys. 79421

[19] Zeiler A et al 1997 Phys. Plasmas 42134

[20] Smolyakov A I 1998 Canadian journal of physics $\mathbf{7 6} 321$

[21] Hinton F L and Horton C W 1971 Phys. Fluids 14116

[22] Chang Z and Callen J D 1992 Phys. Fluids B. 41766 
Gyrofluid potential vorticity equation and turbulent equipartion states

[23] Brizard A Phys. Fluids B 41213

[24] Scott B D 2007 Phys. Plasmas 14102318

[25] Wiesenberger M, Madsen J, and Kendl A 2014 Phys. Plasmas 21092301

[26] Madsen J et al 2011 Phys. Plasmas 18112504

[27] Nycander J and Yankov V V 1996 Physica Scripta T63 174

[28] Isichenko M B and Petviashvili N V 1995 Phys. Plasmas 23650

[29] MATLAB 2014a (8.3.0.532), The MathWorks, Inc., Natick, Massachusetts, United States.

[30] Viezzer E et al 2013 Nucl. Fusion 53053005

[31] Xia H et al 2006 Phys. Rev. Lett. 97255003 